\title{
Using Adaptive Mutation in a GA based Intrusion Detection
}

\author{
S.N.Pawar \\ Asso.Professor (E \&TC), \\ Jawaharlal Nehru Engineering College, \\ Aurangabad, MS, India.
}

\author{
R.S.Bichkar \\ Professor (E \&TC), \\ G.H.Raisoni College of Engineering \& Management, \\ Pune, MS, India.
}

\begin{abstract}
This paper uses a GA-based approach for intrusion detection and uses an adaptive mutation to improve its performance. The drawback of conventional GA is its randomness of mutation which is applied to all the chromosomes irrespective of their fitness. Thus a very good chromosome is equally likely to be disrupted by mutation as a bad one. On the other hand bad chromosomes are less likely to produce good ones through crossover if they are not changed. Hence, it is proposed to use fitness proportionate adaptive mutation in a GA based intrusion detection. This adaptive mutation function does not change the fittest chromosome and causes a change in the low fit chromosomes. This causes the genetic algorithm to arrive at better solution. Experimental results show that this technique improves the fitness of the classification rules and in turn increases the intrusion detection rate.
\end{abstract}

\section{Keywords}

Genetic Algorithms, Intrusion detection, Adaptive mutation.

\section{INTRODUCTION}

Any unauthorized access causing violation to the security policy of a system is called intrusion to a computer. Intrusions cannot be predicted. Hence more focus is put on intrusion detection. The sooner an attack can be detected, the quicker an action can be taken. Intrusion detection can help to collect more information about attacks, strengthening the intrusion prevention methods. Various soft computing techniques such as Genetic Algorithm, Artificial Neural Network and Fuzzy Logic are used to make an intrusion detection system (IDS) smart enough to detect the intrusions at the earliest so that future damage can be avoided.

It is probably impossible to build a completely secure system. The prevention based security philosophy constrains the user's activity and productivity [1]. Hence intrusion detection systems are designed based on various detection techniques, namely Anomaly intrusiondetection and Misuse intrusion detection [2].

\begin{abstract}
Anomaly intrusion detection:
In anomaly IDS the user's behavior is compared with a known standard behavior to detect any significant deviation from normal behavior. This approach can be more effective in protection against unknown or novel attacks since no prior knowledge about specific intrusions is required.
\end{abstract}

However it may cause more false positives because abnormality can be due to a new normal behavior [3].

Misuse intrusion detection:

This is the most widely used IDS. It uses patterns of known attacks or weak spots of the system to identify known intrusions. The signatures and patterns used to identify attacks consist of various options in the packet like source address, destination address, source and destination ports and even the keywords in the content area of a packet.

An IDS can also be classified into two categories based on their location, as host-based and network-based IDS [4].A host-based IDS monitors activities associated with a particular host; whereas a network-based IDS monitors activities associated with network.

This paper proposes an effective GA based approach to generate the classification rules for network intrusion detection. GA is selected because of its robustness, simplicity of operation and power of effect [5]. In conventional GA, the mutationis randomly applied to all the chromosomes irrespective of their fitness. A good chromosome may be disrupted by mutation as a bad one. On the other hand bad chromosomes are less likely to produce good ones through crossover if they are not changed. It used fitness proportionate adaptive mutation [6-10].In adaptive genetic algorithm (AGA), the population converges easily to the locally optimal individuals, because the probabilities of mutation are determined by fitness of chromosomes [11].

Thus far, the motivation of the presented work and the brief overview of the IDS are discussed. The rest of the paper is organized as follows. Section 2 gives an overview of the genetic algorithm employed in this work. In Section 3, survey of the relevant work is made. Section 4 presents the proposed GA based IDS. Section 5 presents the implementation and results and section 6 concludes the paper.

\section{GENETIC ALGORITHM}

Genetic Algorithm (GA) is a technique which works on the mechanics of natural selection. They are based on the Darwin's theory of survival of the fittest. Simplicity of operation and power of effect are the two main attractions of the genetic algorithm approach. They combine survival of the fittest among string structures with a structured yet randomized information exchange to form a search algorithm with some of the innovative flair of human search [5].The major application of GA is in the area of optimization.

The GA process begins with a set of potential solutions or chromosomes which are randomly generated or selected. These chromosomes are normally encoded in the binary form but other forms of encodings are also used. The entire set of these chromosomes comprises a population.

In every generation the fitness of these chromosomes is checked. Fitness function is used to determine the fitness of the chromosomes. Based on the fitness, fittest chromosomes are selected. The chromosomes with poor fitness value are discarded. The selected fit chromosomes undergo crossover and mutation to form a new population. This new population is used for the next generation. Normally, the algorithm 
terminates when either a set number of generations or a satisfactory fitness level has been achieved.

Genetic algorithm is composed of three operators. They are reproduction or selection, crossover or recombination and mutation.

Selection is the process of choosing parents for reproduction. There are many techniques to select the chromosomes, e.g. Roulette wheel selection, Boltzman selection, tournament selection, rank selection, steady state selection etc.

After two parents have been selected by the selection method, crossover takes place. Crossover is an operator that mates two parents (chromosomes) to produce two offspring. Two newborn chromosomes may be better than their parents and the evolution process may continue. The crossover is carried out according to the crossover probability. There are various crossover techniques like one point, two point, uniform etc.

Mutation is a genetic operator that alters one or more gene values in a chromosome. Mutation is carried out according to the mutation probability. It prevents the genetic population from converging to a local minimum. It adds new gene values to the gene pool. These new gene values may cause the genetic algorithm to arrive at better solution. The types of mutation are flip bit, random and min-max etc.

The chromosomes are then evaluated using a certain fitness criteria. When the GA terminates, the chromosome with best fitness is taken as the best solution of the problem.

\section{RELATED WORK USING GA APPROACH}

Different researchers have implemented GA in a different way for intrusion detection.

RenHui Gong et al. (2005) [4] have used a simple genetic algorithm to derive a set of classification rules from network audit data and the support confidence framework is utilized as fitness function to judge the quality of each rule. The generated rules are then used to detect or classify network intrusions in a real time environment.

Chi Hoon Lee et al. (2006) [12] present the novel feature selection method that maximizes class separation between normal and attack patterns of computer network connections. They have focused on selecting a robust feature subset based on the genetic optimization procedure in order to improve a true positive intrusion detection rate.

Nalini N. and RaghavendraRao G. (2006) [13] present a novel method of intrusion detection based on genetic algorithms and principal component analysis. This technique can also be used to detect the class of intrusion. In this paper, they experiment with PCA to reduce the number of features of a TCP connection. This helps in reducing the number of bits required to represent a connection without loss of significant information. They show how network connection information can be modeled as chromosomes and how theparameters in genetic algorithm can be defined in this respect.

Hua Zhou et al. (2007) [14] have used SVM and Genetic Algorithm to increase the classification accuracy. They used GA for feature selection and optimization and then used SVM model to detect intrusions.

Yong Wang et al. (2009) [3] proposed a fitness function, an efficient rule generator for denial of service attack. They used GA toolbox provided by MATLAB (R14) for his implementation. They designed the genetic algorithm using 4 $\mathrm{m}$-files. The rules generated are in if \{condition $\}$ then \{outcome\} form. The rules generated are suitable for continuously changing misuse detection.
Chen Zhongmin et al. (2009) [15] designed a training algorithm model based on abnormality detection. The proposed experimental model is based on a hypothesis that if variable $x$ appears more times than the desired value, there is a possibility of occurrence of abnormality.

Chris Sinclair et al. (2010) [16] have proposed an approach to create rules for an intrusion detection expert system. They employ genetic algorithms and decision trees to automatically generate rules for classifying network connections. They have used genetic algorithms to evolve simple classification rules. The rules are in if \{pattern matched $\}$ then \{generate alert $\}$ form. They have used only five attributes namely source IP address, destination IP address, source IP port, destination IP port, and network protocol. They have used the ID3 algorithm to create decision trees. The final rule generated by decision tree is complete with respect to the training data.

S.N.Pawar and R.S.Bichkar (2012) [17] have used genetic algorithm with enumeration technique. The enumeration technique is used while generating random population. It is used to determine the value of each gene. This enumeration technique substantially reduces the computational time required for population generation and yields more appropriate rules.

Thus, the researchers have implemented GA either to generate the classification rules or to select the appropriate features. Chi Hoon Lee [12] and Hua Zhou et al. [14] have used GA for the selection of appropriate features whereas Gong et al.[4], Yong Wang et al. [3],Chris Sinclair et al. [16] and S.N.Pawarand R.S.Bichkar[17] have used GA for the generation of classification rules. All these researchers have used random mutation which gets applied to all the chromosomes.

\section{THE PROPOSED GA-BASED IDS USING ADAPTIVE MUTATION}

In this work the classification rules are generated using GA approach. These rules are then used to classify or detect the infected connections.

\subsection{Data Set}

MIT Lincoln Laboratory, under Defense Advanced Research Projects Agency (DARPA) and Air Force Research Laboratory (AFRL) sponsorship, has collected and distributed the first standard data for evaluation of computer network intrusion detection systems. This Data is DARPA 1998 data [18]. It consist of tcpdump and BSM list files. Each line in a list file corresponds to a separate session. Each session corresponds to an individual TCP/IP connection between two computers. The first nine columns in list files provide information which identifies the TCP/IP connection.

In this paper, DARPA 1998 data is used for the generation of GA-based rule set and then for the detection of infected connections.

Two subsets from this data set are used: One for training the system and the other to test the detection rate.

\subsection{Feature selection and representation}

Seven most important features of the data set are selected for defining the intrusion rules. These are duration (h: $\mathrm{m}: \mathrm{s}$ ), service (int), source port (int), destination port (int), source IP (a. b. c. d), destination IP (a. b. c. d) and attack name (int).

These features or genes can be represented in different form. They can be encoded in the binary, integer or float. Each of the three parts of the 'Duration' such as hours, minutes, and seconds are encoded in one gene of type byte. The features 'Protocol', 'Source port', 'Destination port' and 'Attack name' are encoded using one gene of type integer [4]. 
The feature 'Source IP' and 'Destination IP' have four octet parts such as $a, b, c$ and $d$. Each part is encoded in one gene of type byte. All the classification rules are in if-then form. All the features except attack name forms a condition ' $A$ ' whereas attack name is the outcome ' $B$ ' of that condition [4].

The wild card values can be used in each of the fields of the rule. In the given implementation,the wild card values are used in the third and fourth octet of both source IP and destination IP. Anumber -1 is put in the field chosen for the wild card.

\subsection{Fitness function}

The fitness function of a rule is decided by checking the number of times it matches with the record connections [4]:

Support $=\mid A$ and $B \mid / N$

Confidence $=\mid A$ and $B|/| A \mid$

Fitness $=w_{1} *$ support $+w_{2} *$ confidence

Where, $N$ is the total number of connections, $|A|$ is the number of connections where the rule matches the portion

of the connection matching the first six features. $\mid A$ and $B \mid$ represent the number of connections that matches both condition part $A$ and outcome part $B$ of the rule. The weights $w_{1}$ and $w_{2}$ can be adjusted to fine tune the algorithm and have used the values of $w_{1}=0.2$ and $w_{2}=0.8$.

\subsection{Crossover and Adaptive Mutation}

Crossover is one of the important steps in GA. There are three types of crossover techniques. They are one-point, two-point and uniform crossover technique. In the given implementation, two point crossover technique is used with a probably Pc.Each gene in each low fit chromosome i.e. the chromosomes having fitness $<0.75$ is checked for possible mutation by generating a random number between zero and one and if this number is less than or equal to the given mutation probability i.e. $\mathrm{Pm}$, then the gene value is changed. Chromosomes having fitness greater than 0.75 are assumed to be the good chromosomes and their mutation probability is kept minimum or zero. This leads to further improvement in the chromosome.

\section{IMPLEMENTATION AND RESULTS}

The genetic algorithm for rule generation is implemented using Java language (JDK6). The front end development environment used is NetBeans7.0. Two subsets were developed from DARPA 1998 data.

Table 1 gives the distributions of record types in both training and testing data set. The first row gives the number of normal network records. The second row gives the distributions of Smurf attack whereas the third row gives the distribution of Neptune attack.

Table 1.The distribution of record types

\begin{tabular}{|c|c|c|}
\hline Record Type & Training Set & Testing Set \\
\hline Normal & 45711 & 4513 \\
\hline Smurf & 524 & 101 \\
\hline Neptune & 15 & 10 \\
\hline
\end{tabular}

The implementation is done in two phases. In the first phase the classification rules are generated using genetic algorithm. Enumeration technique is used while generating the population. Support confidence function is used as fitness function. The GA parameters used were $w_{1}=0.2, w_{2}=0.8$, 2000 generations, population of 300 rules, crossover rate of 0.5 , two-point midsection crossover and mutation rate of 0.01.After generating the classification rules in the first phase, the top ten fittest rules were taken for detection purpose.In the second phase, these rules are used to classify both training as well as testing data set.

Table 2 shows the average fitness of the top ten rules generated for smurf attack and Fig.1 compares the average fitness of the rules generated by conventional algorithm with that of proposed algorithm.

Table 2. Average fitness of the top ten rules for smurf attack

\begin{tabular}{|c|c|c|c|}
\hline $\begin{array}{c}\text { Sr. } \\
\text { No }\end{array}$ & Generations & $\begin{array}{l}\text { Avg. Fitness } \\
\text { (conventional } \\
\text { algorithm) }\end{array}$ & $\begin{array}{l}\text { Avg. fitness } \\
\text { (proposed } \\
\text { algorithm) }\end{array}$ \\
\hline 1 & 500 & 5.51 & 5.63 \\
\hline 2 & 1000 & 6.60 & 6.76 \\
\hline 3 & 1500 & 7.52 & 7.75 \\
\hline 4 & 2000 & 8.12 & 8.40 \\
\hline
\end{tabular}

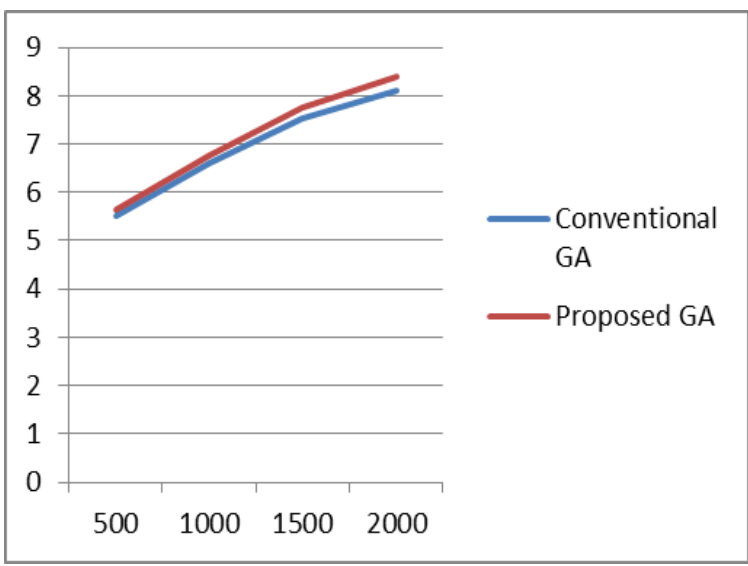

Fig.1 Average fitness comparison over generations

Table 3 shows the percentage detection for different number of generations of GA. As the number of generations is increased, the detection rate is improved. 
Table 3.The Detection rates for different generations

\begin{tabular}{|c|c|c|c|c|}
\hline $\begin{array}{l}\text { Sr. } \\
\text { No }\end{array}$ & $\begin{array}{c}\text { Gener- } \\
\text { ations }\end{array}$ & $\begin{array}{c}\text { Record } \\
\text { Type }\end{array}$ & $\begin{array}{c}\% \\
\text { Detection } \\
\text { (Conv. } \\
\text { Algorithm) }\end{array}$ & $\begin{array}{c}\% \\
\text { Detection } \\
\text { (Proposed } \\
\text { Algorithm) }\end{array}$ \\
\hline \multirow{3}{*}{01} & \multirow{3}{*}{500} & Normal & 74.0 & 74.4 \\
\hline & & Smurf & 76.6 & 77.0 \\
\hline & & Neptune & 81.0 & 81.2 \\
\hline \multirow{3}{*}{02} & \multirow{3}{*}{1000} & Normal & 81.0 & 81.3 \\
\hline & & Smurf & 82.0 & 82.5 \\
\hline & & Neptune & 83.0 & 83.4 \\
\hline \multirow{3}{*}{03} & \multirow{3}{*}{1500} & Normal & 91.0 & 91.7 \\
\hline & & Smurf & 92.2 & 92.6 \\
\hline & & Neptune & 96.0 & 96.4 \\
\hline \multirow{3}{*}{04} & \multirow{3}{*}{2000} & Normal & 98.2 & 98.6 \\
\hline & & Smurf & 96.0 & 97.3 \\
\hline & & Neptune & 100.0 & 100.0 \\
\hline
\end{tabular}

From table 3 it is evident that the results obtainedby proposed approach are slightly better than those obtained by earlier approach [17].

\section{CONCLUSION}

In this paper, an effective technique of using adaptive mutation in a GA based intrusion detection is presented. The system is implemented in two steps. In the first step, GA is used to generate classification rules where as in the second step these rules are used for intrusion detection.

From the results presented it is evident that average fitness of the rules generated by proposed algorithm is better than the conventional algorithm. The cause of this improvement is adaptive mutation that does not disrupt the fit chromosomes and causes change in only less fit chromosomes giving them a chance for improvement.It is also evident from the results that the rules generated by the proposed algorithm give better detection results as compared to the earlier approach

\section{REFERENCES}

[1] A.Vesely, D.Brechlerova, "Neural Networks in Intrusion Detection Systems", AGRIC.ECON.CZECH, 50, 2004 (1):35-39.

[2] S.Owais, V.Snasel and P.Kromer, A. Abraham, "Survey Using Genetic Algorithm Approach in Intrusion Detection Systems Techniques", $7^{\text {th }}$ Computer Information Systems and Industrial Management Applications, 2008, IEEE press, June2008, pp.300-307.
[3] Yong Wang, DawuGu, XiuxiaTian, Jing Li, "Genetic Algorithm Rule Definition for Denial of Services Network Intrusion Detection", International Conference on Computational Intelligence and Natural Computing, IEEE, 2009, pp.99-102.

[4] RenHui Gong, Mohammad Zulkernine, PurangAbolmaesumi, "A Software Implementation of a Genetic Algorithm Based Approach to Network Intrusion Detection", SNPD/SAWN'05, IEEE, 2005.

[5] Biswanath Mukherjee, L.ToddHerbe- rlein and Karl N. Levitt, "Network Intrusion Detection", IEEE Network, 8(3):26-41, May/June 1994.

[6] S.MarsiliLibelli, P.Alba, "Adaptive Mutation in Genetic Algorithms", Soft Computing 4 (2000), Springer- Verlog, 2000, pp.76-80.

[7] XuHaiyan, "Research for New Modified Adaptive Genetic Algorithm," World Automation Congress, IEEE, 2012,pp.1- 4.

[8] Dirk Thierens, "Adaptive MutationRate Control Schemes in Genetic Algorithms”, IEEE, 2002,pp.980-985.

[9] C.W. Ho, K.H. Lee and K.S. Leung, "A Genetic Algorithm Based on Mutation and Crossover with Adaptive Probabilities" IEEE,1999, pp.768-785.

[10] FatemehVafaee and Peter C. Nelson , "A Genetic Algorithm That Incorporates an Adaptive Mutation Based On an Evolutionary Model", International Conference on Machine Learning and Applications,IEEE,2009,pp.101107.

[11] Zhiming Liu, Jiliu Zhou, Su Lai, "New Adaptive Genetic Algorithm Based on Ranking," Proceedings of the Second International Conference on Machine Learning and Cybernetics, X.'an, 2-5 November 2003. IEEE, 2003, pp.1841-1844.

[12] ChiHoon Lee, Sung Woo Shin and Jin Wook Chung, "Network Intrusion Detection Through Genetic Feature Selection", SNPD, IEEE, 2006.

[13] Nalini N. and RaghavendraRao G., "Network Intrusion Detection via a Hybrid of Genetic Algorithms and Principal Component Analysis", IEEE, 2006.

[14] Hua Zhou, Xingu Meng and Li Zhang, "Application of Support Vector Machine and Genetic Algorithm to Network Intrusion Detection”, IEEE, 2007.

[15] Chen Zhongmin, FengJianyuan, Xu Sheng, XuRenzuo, "The Research of Intrusion Detection Technology Based on Genetic Algorithms," International Conference on Networks Security, Wireless Communications and Trusted Computing, IEEE, 2009.

[16] Sinclair Chris, L. Pierce, and S. Matzner, "An Application of Machine Learning to Network Intrusion Detection", Annual Computer Security Applications Conference, 1999, pp. 371-377.

[17] S. N. Pawar and R. S. Bichkar, "UsingEnume-ration in a GA based Intrusion Detection", International Journal of Computer Applications (IJCA), October, 2012.

[18] MIT Lincoln Laboratory, DARPA datasets,MIT,USA,http://www.ll.mit.edu/mission/commu nications/ist/corpora/ideval/data/1998data.html 